\title{
Low levels of serum vitamin D3 are associated with fibromyalgia syndrome in pre-menopausal women: A pilot study
}

\author{
Nezihe Akar (D, Nil Sayıner Çaglar (D), Ebru Aytekin (D), Abdullah Akar (D), Özge Aksu (D), Nuran Öz (D) \\ Department of Physical Medicine and Rehabilitation, Health Sciences University, Istanbul Training and Research Hospital, Istanbul, Turkey
}

Received: March 20, 2018 Accepted: November 05, 2018 Published online: March 03, 2020

\section{ABSTRACT}

Objectives: This study aims to investigate the prevalence of fibromyalgia syndrome (FMS) among patients with low and adequate vitamin D levels in premenopausal women.

Patients and methods: Between October 2012 and April 2013, a total of 80 premenopausal patients (mean age $38.1 \pm 7.1$ years; range, 18 to 50 years) with non-specific musculoskeletal symptoms were included in the study. The determination of 25-hydroxyvitamin D3 (25(OH)D3) deficiency was based on a reference value of $25 \mathrm{ng} / \mathrm{mL}$. Patients with deficient serum 25(OH)D3 levels $(<25 \mathrm{ng} / \mathrm{mL}$, $\mathrm{n}=40)$ comprised the patient group, while those with adequate serum $25(\mathrm{OH}) \mathrm{D} 3$ levels $(\geq 25 \mathrm{ng} / \mathrm{mL}, \mathrm{n}=40)$ comprised the control group. Data including demographic characteristics, laboratory parameters (i.e., calcium, phosphorus, alkaline phosphatase [ALP], and parathyroid hormone $[\mathrm{PTH}]$ ), and clinical findings (i.e., proximal muscle weakness [PMW] and periarticular sensitivity [PAS]) were recorded and compared between the groups. Pain severity was evaluated using the visual analog scale (VAS), psychological status was evaluated using the Beck Depression Inventory (BDI), and quality of life (QoL) was evaluated using the Short Form 36 (SF-36). The diagnosis of FMS was made based on the 2010 criteria of the American College of Rheumatology.

Results: Although there were no statistically significant differences in the demographic characteristics between the groups, ALP and PTH levels were higher in the patient group $(\mathrm{p}<0.05)$. The VAS, frequency of FMS, PMW, and PAS were also significantly higher in the patient group $(\mathrm{p}<0.05)$. Depression and the mental and physical component scores of the SF-36 did not significantly differ between the two groups $(\mathrm{p}<0.05)$.

Conclusion: Our study results suggest that patients with low 25(OH)D3 levels may more frequently experience FMS and pain than healthy individuals.

Keywords: Depression, fibromyalgia syndrome, pain, quality of life, vitamin D.

Vitamin $\mathrm{D}$ is a steroid hormone which plays a critical role in maintaining the equilibrium of calcium (Ca) and phosphorus (P) levels in the body as well as bone health. ${ }^{[1]}$ It is a fat-soluble vitamin present in many foods, but also endogenously produced by the skin when exposed to ultraviolet rays from sunlight. Endogenous synthesis also involves the kidney and liver, as the sites of vitamin D hydroxylation to 1,25 hydroxyvitamin $\mathrm{D}$ and 25 -hydroxyvitamin $\mathrm{D}$, respectively. ${ }^{[2]}$

Many factors affect serum 25-hydroxyvitamin D3 $(25(\mathrm{OH}) \mathrm{D} 3)$ levels including age, skin pigmentation, reduced or restricted sun exposure (e.g., homebound persons, individuals covering their skin for cultural/ religious reasons), eating habits, season, and reduced cutaneous synthesis (e.g., in the elderly). In addition, those with malabsorption syndrome and obesity are also at risk for vitamin D deficiency. ${ }^{[2-4]}$ Since vitamin $\mathrm{D}$ receptors are expressed on almost all cells, ${ }^{[2,5]}$ vitamin $\mathrm{D}$ deficiency can occur in association with many diseases including musculoskeletal disorders (fibromyalgia, fibromyalgia syndrome [FMS]), neuromuscular disorders, infections, autoimmune diseases, lung diseases, metabolic syndrome, diabetes,

Corresponding author: Ebru Aytekin, MD. SBÜ İstanbul Eğitim ve Araştırma Hastanesi Fiziksel Tip ve Rehabilitasyon Kliniği, 34098 Fatih, İstanbul, Türkiye. 
cardiovascular diseases, cognitive function disorders, psychiatric conditions, and many types of cancer. ${ }^{[1,6]}$

Fibromyalgia syndrome is a chronic syndrome with an increasing prevalence, characterized by widespread musculoskeletal pain in combination with a variety of cognitive symptoms and fatigue. ${ }^{[5]}$ The complaints of patients with FMS resemble in many aspects those of patients with vitamin D deficiency and may overlap. The association between FMS and vitamin D deficiency is still controversial. ${ }^{[6]}$ Some authors have reported a significant correlation between FMS and vitamin $\mathrm{D}$ deficiency, whereas some others have found no correlation..$^{[1]}$

The primary aim of the present study was to investigate the prevalence of FMS among patients with low and adequate vitamin D levels in premenopausal women. The secondary aim was to analyze the effects of vitamin D on pain, depression, and quality of life (QoL) in those patients.

\section{PATIENTS AND METHODS}

Between October 2012 and April 2013, a total

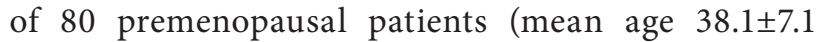
years; range, 18 to 50 years) who were admitted to Istanbul Training and Research Hospital Physical Medicine and Rehabilitation outpatient clinic with non-specific musculoskeletal pain and whose $\mathrm{Ca}, \mathrm{P}$, alkaline phosphatase (ALP), parathyroid hormone $(\mathrm{PTH})$, and 25(OH)D3 levels were measured were included in the study. The patient group consisted of 40 patients with low serum $25(\mathrm{OH}) \mathrm{D} 3$ levels $(<25 \mathrm{ng} / \mathrm{mL})$, and the control group comprised 40 patients whose serum 25(OH)D3 levels were within normal limits $(\geq 25 \mathrm{ng} / \mathrm{mL}$ ). Those with systemic, metabolic, endocrine, tumoral, infectious, or neurological diseases were excluded from the study. Postmenopausal women, pregnant women, those with major psychiatric illness, those taking drugs affecting serum 25(OH)D3, ALP, PTH, Ca, or P levels and those administered $\mathrm{Ca}$ or vitamin $\mathrm{D}$ supplements within the past three months were also excluded. A written informed consent was obtained from each participant. The study protocol was approved by the Istanbul Training and Research Hospital Ethics Committee. The study was conducted in accordance with the principles of the Declaration of Helsinki.

Data including demographic characteristics of the patients (i.e., age, weight, height, body mass index [BMI], education level, menarche age, number of pregnancies, lactation duration, skin color, smoking, use of antidepressant medication or an antiepileptic drug, and previous bone fracture) were recorded. All patients underwent a complete physical examination including periarticular sensitivity (PAS). Manual muscle testing for proximal muscle weakness (PMW) and observational gait analysis were also performed.

Pain severity was evaluated using the visual analog scale (VAS). All patients were requested to indicate pain severity based on a $10-\mathrm{cm}$ scale ranging from "No pain" to "Extreme pain". Using the VAS, higher scores indicate increased severity. Psychological status was also assessed using the 21-item Beck Depression Inventory (BDI), which is used to calculate the risk for depression and to measure the levels of depressive symptoms. A value of $0-3$ is assigned for each answer to each item. The adaptation, reliability, and validity of the BDI in the Turkish population have been conducted. ${ }^{[7]}$

The QoL was evaluated using the Turkish version of the QoL questionnaire of the Short Form 36 (SF-36) ${ }^{[8]}$ It is a self-rated form and includes a total of 36 questions with eight different sub-dimensions related to health: physical function, social function, role limitations due to physical problems and emotional problems, mental health, vitality, bodily pain, and general health perception. For each dimension, item scores are scored from 0 (worst health status) to 100 (best health status) and transformed into a scale.

The diagnosis of FMS was made based on the 2010 criteria of the American College of Rheumatology, which consists of the widespread pain index (WPI) and the symptom severity (SS) scale. The diagnostic criteria for FM are satisfied, if the following three conditions are met: WPI $\geq 7$ and SS score $\geq 5$ or a WPI of 3-6 and a SS score $\geq 9$; the presence of symptoms at a similar level for at least three months; and the absence of a disorder that would otherwise explain the pain. Demographic characteristics, laboratory parameters, physical examination findings, pain levels, depression frequency, FMS presence, and QoL were compared between the patient and control groups.

\section{Statistical analysis}

Statistical analysis was performed using the Statistical Package for the Social Sciences (SPSS) version 15.0 software (SPSS Inc., Chicago, IL, USA). Descriptive data were presented in mean \pm standard deviation (SD) for normally distributed variables and in median (min-max) for non-normally distributed variables. Categorical data were expressed in number and percentage. The two groups were compared 


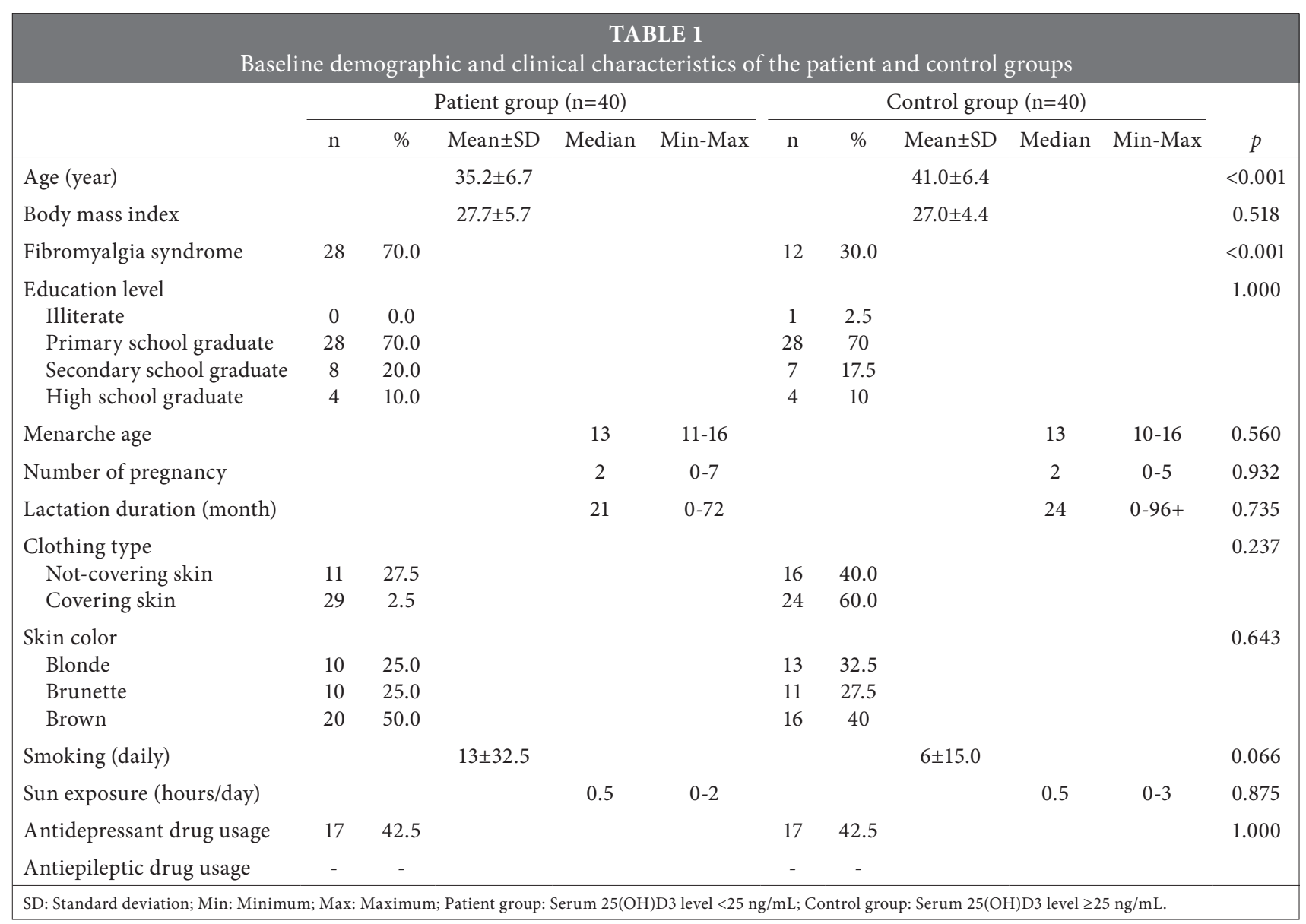

using the Student's t-test if the numerical data were normally distributed, and the Mann-Whitney U test was used for non-normally distributed variables. The chi-square test was used to examine relationships between categorical variables. A $p$ value of $<0.05$ was considered statistically significant.

\section{RESULTS}

Baseline demographic and clinical characteristics of the patient (40 patients with serum 25(OH)D3 levels $<25 \mathrm{ng} / \mathrm{mL}$ ) and control groups (40 patients with serum $25(\mathrm{OH}) \mathrm{D} 3$ levels $\geq 25 \mathrm{ng} / \mathrm{mL}$ ) are shown in Table 1. There was no significant difference in the demographic and clinical characteristics between the patient and control groups ( $\mathrm{p}>0.05)$.

The laboratory parameters of the groups are shown in Table 2. The mean serum 25(OH)D3 levels in the patients and controls were $12.2 \pm 6.3 \mathrm{ng} / \mathrm{mL}$ and $50.5 \pm 30.3 \mathrm{ng} / \mathrm{mL}$, respectively. The frequency of FMS was $70 \%$ in the patient group and $30 \%$ in the

\begin{tabular}{|c|c|c|c|c|c|}
\hline \multicolumn{6}{|c|}{$\begin{array}{l}\text { TABLE } 2 \\
\text { Laboratory parameters of the patient and control groups }\end{array}$} \\
\hline & \multicolumn{2}{|c|}{ Patient group $(n=40)$} & \multicolumn{2}{|c|}{ Control group $(n=40)$} & \multirow[b]{2}{*}{$p$} \\
\hline & Median & Min-Max & Median & Min-Max & \\
\hline Calcium & 9 & $8-10$ & 9 & $8-10$ & 0.477 \\
\hline Phosphorus & 3 & $2-4$ & 3 & $2-4$ & 0.787 \\
\hline Alkaline phosphatase & 61 & $36-153$ & 55 & $29-82$ & 0.040 \\
\hline Parathyroid hormone & 61 & $15-309$ & 48 & $16-96$ & 0.006 \\
\hline
\end{tabular}




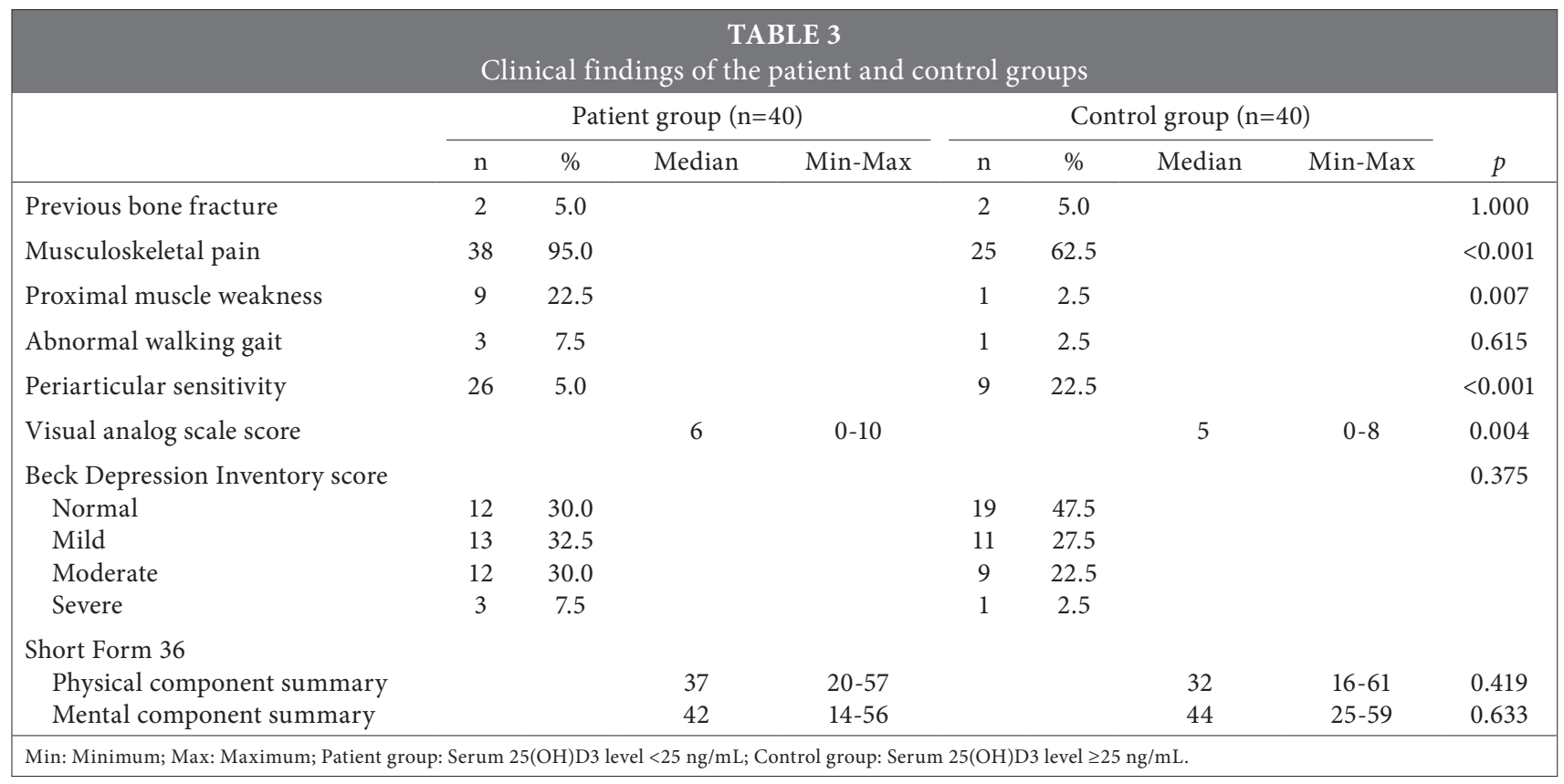

control group, indicating a statistically significant difference $(\mathrm{p}<0.001)$. In addition, the mean ALP and PTH values were significantly higher in the patient group than controls $(p<0.05)$. However, there was no significant difference in the mean $\mathrm{Ca}$ and $\mathrm{P}$ levels between the groups.

The mean BDI previous bone fracture, abnormal walking gait, mental and physical component scores of the SF-36 did not significantly differ between the groups $(\mathrm{p}<0.05)$, while the mean VAS scores, PMW, and PAS were found to be significantly higher in the patient group than the control group $(\mathrm{p}<0.05)$ (Table 3$)$.

\section{DISCUSSION}

In this study, patients with low 25(OH)D3 levels had a higher frequency of FMS than those with normal $25(\mathrm{OH}) \mathrm{D} 3$ levels $(70 \%$ vs. $28 \%)$. In addition, these patients had higher pain levels, although no significant difference was found in depression and QoL. In the current study, premenopausal women were chosen, as they are about nine times more likely to develop FMS than men and, in postmenopausal women, pain due to osteoarthritis rather than non-specific widespread musculoskeletal pain is more common. ${ }^{[6,9]}$

Vitamin D plays a key role in health and the QoL. It is supplied by cutaneous synthesis and is dependent on sunlight exposure and dietary intake. Vitamin D deficiency is a widespread global problem. ${ }^{[2]} \mathrm{A}$ German health and examination survey of adults showed that slightly more than $60 \%$ of the German population has 25(OH)D3 levels below $20 \mathrm{ng} / \mathrm{mL}$, particularly during winter and spring seasons, due to inadequate ultraviolet $\mathrm{B}$ radiation (at a wavelength of 290 to $315 \mathrm{~nm}$ ), and particularly in individuals living at higher altitudes ${ }^{[10]}$ However, higher BMI, lack of sports activity, and high media use were found to be potentially modifiable factors of poorer vitamin $\mathrm{D}$ status. In addition, abnormalities in vitamin $\mathrm{D}$ metabolism were reported in Muslim women clothing much of their skin. ${ }^{[1]}$ In patients with chronic pain/FMS, low 25(OH)D levels were found to occur more often than in other general rheumatology outpatients. ${ }^{[12]}$ Platnikof and Quigley ${ }^{[13]}$ reported that $89 \%$ of patients with chronic musculoskeletal pain had 25(OH)D3 deficiency. In contrast, in the series of Warner and Arnspiger, ${ }^{[14]}$ low vitamin D levels were not associated with diffuse musculoskeletal pain, and treatment with vitamin $\mathrm{D}$ did not reduce pain in patients with diffuse pain with low vitamin D levels. In FMS, whether there is a causal relationship with hypovitaminosis D or whether hypovitaminosis is a risk factor for a worsening of FMS through peripheral and central mechanisms is unknown. Nonetheless, treating vitamin $\mathrm{D}$ deficiency can benefit long-term bone health and muscle strength, both of which are important not only for patients with FMS, but also for the general population. ${ }^{[6]}$ In our study, pain/FMS negatively correlated with low serum 25(OH)D3 levels. However, it is unclear whether vitamin D 
deficiency in our patients with FMS could develop secondarily, as a result of sedentary lifestyle, limited social and outdoor activities, and poor eating habits.

Vitamin D deficiency causes muscle weakness and muscle aches and pain in both children and adults. Vitamin D interacts with the nuclear receptors in the muscle tissue to increase muscle strength. ${ }^{[15,16]}$ Bischoff et al. ${ }^{[17]}$ found that adults with vitamin D deficiency suffered from muscle weakness and were more likely to fall. In our study, PMW and PAS were also found to be significantly higher in the patients than the control group.

Systematic reviews and meta-analyses of population-based, cross-sectional, prospective cohort studies have shown that low serum 25(OH)D3 levels are associated with depressive symptoms and clinical depression. ${ }^{[18,19]}$ To date, several risk factors for vitamin $\mathrm{D}$ deficiency have been identified, some of which are also associated with depression. von Känel et al. ${ }^{[20]}$ investigated 380 patients including $70 \%$ females (mean age $47 \pm 12$ years) who were consecutively hospitalized with the definitive diagnosis of an ICD-10 depressive episode. The authors found that patients with vitamin D deficiency scored higher on the Hospital Anxiety and Depression Scale and on the anhedonia symptom factor survey than those with inadequate $(\mathrm{p} \leq 0.023)$ or adequate $(\mathrm{p} \leq 0.008)$ serum $25(\mathrm{OH}) \mathrm{D} 3$ levels. Based on these findings, they concluded that vitamin D deficiency was significantly associated with increased levels of depressive symptoms in patients hospitalized with a depressive episode. Vitamin D modulates the hypothalamic-pituitary-adrenal axis, regulating adrenaline, noradrenaline, and dopamine production through vitamin $\mathrm{D}$ receptors in the adrenal cortex. It also protects against the depletion of dopamine and serotonin centrally. Daily supplementation with at least $800 \mathrm{IU}$ vitamin $\mathrm{D}$ improves depression to a similar extent as antidepressant medications. ${ }^{[21]}$ Moreover, vitamin D supplementation may augment the effects of antidepressants in patients with major depressive disorder. In our study, the depression rate was higher in patients than in the control group. However, the rate of antidepressant drug use was high in both groups, which might have precluded a full examination of the association between depression and 25(OH)D3 levels.

In the literature, there is little information describing the QoL, consequences for daily living, or psychosocial status of patients who meet the diagnostic criteria for FMS. Cöster et al. ${ }^{[22]}$ showed that daily living activities were more adversely affected in patients with FMS. ${ }^{[22]}$ However, Yilmaz et al. ${ }^{[1]}$ found that vitamin D replacement improved the QoL in patients with chronic, non-specific, widespread musculoskeletal pain. Similarly, Wepner et al. ${ }^{[23]}$ reported that vitamin $\mathrm{D}$ replacement improved the QoL in FMS patients. Nonetheless, our study provided no evidence of such a relationship between vitamin $\mathrm{D}$ insufficiency and QoL.

There are several limitations to the present study. Although we considered sun exposure, clothing type, skin color, and cigarette use, we were unable to asses other variables such as season, eating habits, sports activity, and media use. In addition, only premenopausal female patients were included and, thus, the results of this investigation cannot be extrapolated to the opposite sex. Finally, we were unable to re-evaluate the control group after maintenance treatment of vitamin D.

In conclusion, our study results show that low 25(OH)D3 levels are associated with pain and FMS, despite the lack of an apparent effect on QoL. Based on these findings, we can speculate that patients with low 25(OH)D3 levels may more frequently experience FMS and pain than healthy individuals. However, further vitamin $\mathrm{D}$ supplementation trials evaluating different regimens and doses are needed to identify whether improving serum 25(OH)D3 levels has beneficial effects on pain, depression, and QoL in patients with FMS.

\section{Declaration of conflicting interests}

The authors declared no conflicts of interest with respect to the authorship and/or publication of this article.

\section{Funding}

The authors received no financial support for the research and/or authorship of this article.

\section{REFERENCES}

1. Yilmaz R, Salli A, Cingoz HT, Kucuksen S, Ugurlu H. Efficacy of vitamin D replacement therapy on patients with chronic nonspecific widespread musculoskeletal pain with vitamin D deficiency. Int J Rheum Dis 2016;19:1255-62.

2. Wintermeyer E, Ihle C, Ehnert S, Stöckle U, Ochs G, de Zwart P, et al. Crucial Role of Vitamin D in the Musculoskeletal System. Nutrients 2016;8. pii: E319.

3. Knutsen KV, Brekke M, Gjelstad S, Lagerløv P. Vitamin D status in patients with musculoskeletal pain, fatigue and headache: a cross-sectional descriptive study in a multiethnic general practice in Norway. Scand J Prim Health Care 2010;28:166-71.

4. Shinchuk LM, Holick MF. Vitamin D and rehabilitation: improving functional outcomes. Nutr Clin Pract 2007;22:297-304. 
5. Karras S, Rapti E, Matsoukas S, Kotsa K. Vitamin D in Fibromyalgia: A Causative or Confounding Biological Interplay? Nutrients. 2016 Jun 4;8(6). pii: E343.

6. Jesus CA, Feder D, Peres MF. The role of vitamin D in pathophysiology and treatment of fibromyalgia. Curr Pain Headache Rep 2013;17:355.

7. Hisli N. A study on the validity of Beck Depression Scale. J Psychol 1988;6:118-26.

8. Koçyiğit H, Aydemir Ö, Fişek G, Ölmez N, Memiş A. Reliability and validity of Turkish version of Short Form 36: A study of patients with rheumatoid disorder. J Drug Ther 1999;12:102-6.

9. Okumus M, Koybası M, Tuncay F, Ceceli E, Ayhan F, Yorgancioglu R, et al. Fibromyalgia syndrome: is it related to vitamin $\mathrm{D}$ deficiency in premenopausal female patients? Pain Manag Nurs 2013;14:e156-e63.

10. Rabenberg M, Scheidt-Nave C, Busch MA, Rieckmann N, Hintzpeter B, Mensink GB. Vitamin D status among adults in Germany--results from the German Health Interview and Examination Survey for Adults (DEGS1). BMC Public Health 2015;15:641.

11. el-Sonbaty MR, Abdul-Ghaffar NU. Vitamin D deficiency in veiled Kuwaiti women. Eur J Clin Nutr 1996;50:315-8.

12. Tandeter H, Grynbaum M, Zuili I, Shany S, Shvartzman P. Serum 25-OH vitamin D levels in patients with fibromyalgia. Isr Med Assoc J 2009;11:339-42.

13. Plotnikoff GA, Quigley JM. Prevalence of severe hypovitaminosis $\mathrm{D}$ in patients with persistent, nonspecific musculoskeletal pain. Mayo Clin Proc 2003;78:1463-70.

14. Warner AE, Arnspiger SA. Diffuse musculoskeletal pain is not associated with low vitamin D levels or improved by treatment with vitamin D. J Clin Rheumatol 2008;14:12-6.
15. Holick MF. Vitamin D deficiency: what a pain it is. Mayo Clin Proc 2003;78:1457-9.

16. Glerup H, Mikkelsen K, Poulsen L, Hass E, Overbeck S, Thomsen J, et al. Commonly recommended daily intake of vitamin D is not sufficient if sunlight exposure is limited. J Intern Med 2000;247:260-8.

17. Bischoff HA, Stähelin HB, Dick W, Akos R, Knecht M, Salis C, et al. Effects of vitamin D and calcium supplementation on falls: a randomized controlled trial. J Bone Miner Res 2003;18:343-51.

18. Ju SY, Lee YJ, Jeong SN. Serum 25-hydroxyvitamin D levels and the risk of depression: a systematic review and metaanalysis. J Nutr Health Aging 2013;17:447-55.

19. Anglin RE, Samaan Z, Walter SD, McDonald SD. Vitamin $\mathrm{D}$ deficiency and depression in adults: systematic review and meta-analysis. Br J Psychiatry 2013;202:100-7.

20. von Känel R, Fardad N, Steurer N, Horak N, Hindermann E, Fischer F, et al. Vitamin D Deficiency and Depressive Symptomatology in Psychiatric Patients Hospitalized with a Current Depressive Episode: A Factor Analytic Study. PLoS One 2015;10:e138550.

21. Spedding S. Vitamin D and depression: a systematic review and meta-analysis comparing studies with and without biological flaws. Nutrients 2014;6:1501-18.

22. Cöster L, Kendall S, Gerdle B, Henriksson C, Henriksson KG, Bengtsson A. Chronic widespread musculoskeletal pain - a comparison of those who meet criteria for fibromyalgia and those who do not. Eur J Pain 2008;12:600-10.

23. Wepner F, Scheuer R, Schuetz-Wieser B, Machacek P, Pieler-Bruha E, Cross HS, et al. Effects of vitamin D on patients with fibromyalgia syndrome: a randomized placebo-controlled trial. Pain 2014;155:261-8. 